\title{
Alternative BSE Risk Assessment Methodology for Beef and Beef Offal Imported into Japan
}

\author{
Yasuhiro YOSHIKAWA ${ }^{1) *}$, Motohiro HORIUCHI ${ }^{2}$, Naotaka ISHIGURO ${ }^{3)}$, Mutsuyo KADOHIRA ${ }^{4)}$, Satoshi $\mathrm{KAI}^{5}$, \\ Hidehiro MIZUSAWA $^{6}$, Chisato NAGATA ${ }^{7)}$, Takashi ONODERA ${ }^{8)}$, Tetsutaro SATA ${ }^{9)}$, Toshiyuki TSUTSUI ${ }^{10)}$, \\ Masahito YAMADA ${ }^{11)}$ and Shigeki YAMAMOTO ${ }^{12)}$ \\ 1) School of Veterinary Medicine, Kitasato University, 23-35-1 Higashi, Towada, Aomori 034-8628, Japan \\ ${ }^{2)}$ Laboratory of Veterinary Hygiene, Department of Applied Veterinary Sciences, Graduate School of Veterinary Medicine, Hokkaido \\ University, Sapporo, Hokkaido 060-0818, Japan \\ 3) Laboratory of Food and Environmental Hygiene, Department of Veterinary Medicine, Faculty of Applied Biological Sciences, Gifu \\ University, Gifu 501-1193, Japan \\ 4) Department of Life Science and Agriculture, Obihiro University of Agriculture and Veterinary Medicine, Obihiro, Hokkaido 080-8555, Japan \\ ${ }^{5)}$ Faculty of Business, Marketing and Distribution, Nakamura Gakuen University, Fukuoka, Fukuoka 814-0198, Japan \\ 6) Department of Neurology and Neurological Science, Tokyo Medical and Dental University, Yushima, Bunkyo-ku, Tokyo 113-8619, Japan \\ ${ }^{7)}$ Department of Epidemiology \& Preventive Medicine, Graduate School of Medicine, Gifu University, Gifu 501-1193, Japan \\ ${ }^{8)}$ Graduate School of Agricultural and Life Sciences, The University of Tokyo, 1-1-1 Yayoi, Bunkyo-ku, Tokyo 113-8657, Japan \\ 9) Department of Pathology, National Institute of Infectious Diseases, Toyama, Shinjuku-ku, Tokyo 162-8640, Japan \\ ${ }^{10)}$ Epidemiological Research Team, National Institute of Animal Health, Tsukuba, Ibaraki 305-0856, Japan \\ ${ }^{11)}$ Department of Neurology and Neurobiology of Aging, Graduate School of Medical Science, Kanazawa University, Kanazawa \\ 920-8640, Japan \\ ${ }^{12)}$ Division of Biomedical Food Research, National Institute of Health, Yoga, Setagaya-ku, Tokyo 158-8501, Japan
}

(Received 11 September 2010/Accepted 31 October 2011/Published online in J-STAGE 14 November 2011)

ABSTRACT. The Food Safety Commission (FSC) of Japan, established in July 2003, has its own initiative to conduct risk assessments on food stuffs known as "self-tasking assessment". Within this framework, the FSC decided to conduct a risk assessment of beef and beef offal imported into Japan from countries with no previous BSE reports; thus, a methodology was formed to suit to this purpose. This methodology was partly based on the previous assessments of Japanese domestic beef and beef imported from U.S.A./Canada, but some modifications were made. Other organizations' assessment methods, such as those used for BSE status assessment in live cattle by the OIE and EFSA's GBR, were also consulted. In this review, the authors introduce this alternative methodology, which reflects (1) the risk of live cattle in the assessed country including temporal risks of BSE invasion and domestic propagation, with the assessment results verified by surveillance data, and (2) the risk of beef and beef offal consisting of cumulative BSE risk by types of slaughtering and meat production processes implemented and the status of mechanically recovered meat production. Other possible influencing factors such as atypical BSE cases were also reviewed. The key characteristic of the current assessment is a combination of the time-sequential risk level of live cattle and qualitative risk level of meat production at present in an assessed country.

KEY WORDS: beef, BSE, importation, prion diseases, risk assessment.

doi: 10.1292/jvms.10-0393; J. Vet. Med. Sci. 74(8): 959-968, 2012

More than 20 years have passed since BSE was officially recognized in the U.K. Now, there is prominent evidence showing the efficacy of a real feed ban and the abolishment of using meat and bone meal (MBM) derived from mammals in feeds for mammals. The total number of BSEpositive cases in the world last year was less than that of one day when the BSE outbreak was at its peak in the U.K. from 1992 through 1993. However, the U.K. continued to

*Correspondence to: Yoshikawa, Y., School of Veterinary Medicine, Kitasato University, 23-35-1 Higashi, Towada, Aomori 034-8628, Japan.

e-mail: ayyoshi@mail.ecc.u-tokyo.ac.jp or yyoshikawa@cis.ac.jp Authors' notes: The authors, except for the first author, are listed alphabetically. This article is based on the discussion at a Prion Expert Committee meeting.

C2012 The Japanese Society of Veterinary Science spread the sources of BSE pathogens, such as live cattle and animal feeds, to two dozen countries, resulting in a cumulative number of more than 220 variant CJD patients in the world [9].

Currently, Japan imports beef and beef offal from the U.S.A. and Canada, two countries that have previously experienced BSE cases and for which the Food Safety Commission (FSC) in Japan has already assessed the BSE risks of their beef and beef offal. Besides these two countries, Japan also imports beef and beef offal from other countries where no BSE cases have been reported so far. However, some of these countries were categorized as Geographical BSE Risk (GBR) category III by the European Food Safety Agency (EFSA). According to EFSA's definition, countries are designated as GBR category III either because they are estimated to have a reasonably high possibility of having BSE cases that have not been detected or because they 


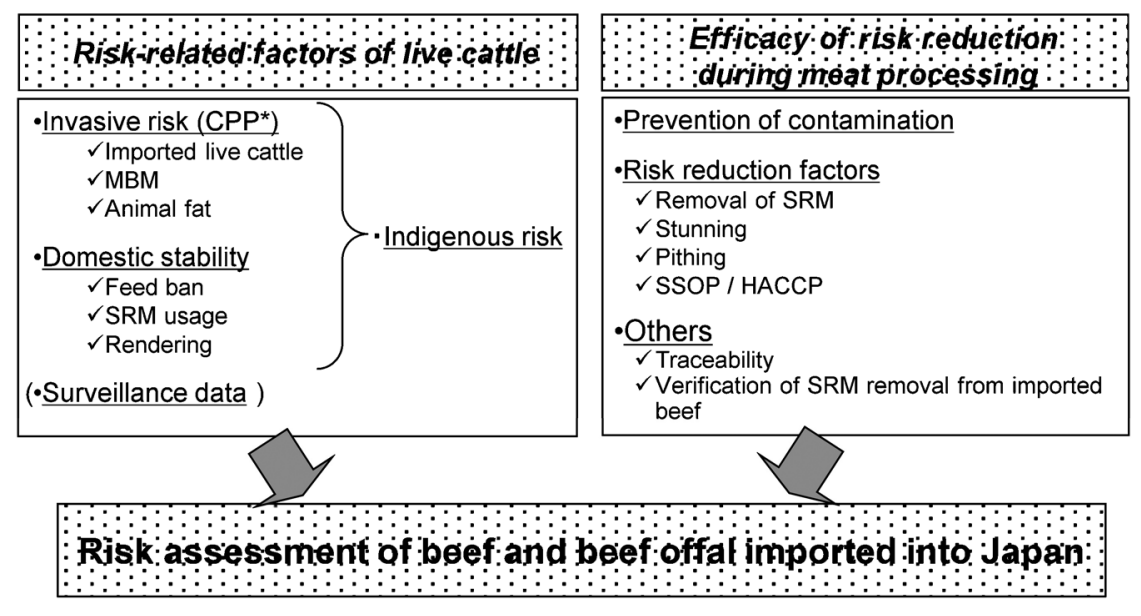

Fig. 1. Schematic presentation of the risk assessment methodology employed by the PEC for the current assessment. Briefly, the risk-related factors in live cattle (shown in the left box) were combined with the risk-reducing efficacy at meat processing facilities (shown in the right box) to gain the total risk assessment targeting beef and beef offal imported into Japan. *: Contamination probability points.

have had a few confirmed cases of BSE. Among exporters to Japan, there are also countries that have simply not been assessed by EFSA's GBR.

Japanese risk managers presently request importers of beef and beef offal from the above countries to submit official health certificates confirming that the cattle are of healthy origin and also ask that they refrain from importing specified risk materials (SRM). Although the health certificates are confirmed at quarantine stations, there are currently no measures to clarify the exclusion of SRM among beef products imported. There is also uncertainty over potential risks of imported beef and beef offal due to insufficient availability of data related to BSE prevalence and anti-BSE countermeasures in the above-mentioned countries.

The FSC in Japan conducts risk assessments at the request of risk managers, or alternatively, it can also conduct assessments on its own initiative, termed "self-tasking assessment". The process of hazard selection for self-tasking assessment is as follows. The Expert Committee for Planning collects information and screens the possible assessment subjects based on the degree of public concern in Japan, based on demands for information collection either due to increasing necessity for developing hazards or based on items that are heavily requested for assessment. Selected subjects are then discussed for potential assessment at the Commission's opinion exchange meetings, and finally, the FSC officially adopts the hazards of choice to be the next subject of selftasking assessment.

Risk assessment of beef and beef offal imported into Japan was among the most requested items during public meetings and other occasions hosted by the FSC. Behind these requests, there seemed to be public concern over uncertainty about BSE risks in beef and beef products imported from countries other than the U.S.A. and Canada. With this situation, the FSC decided to conduct "risk assessment of beef and beef offal imported into Japan" as its self-tasking assessment.

The current assessment conducted by the Prion Expert Committee (PEC) of the FSC in Japan is based on the following concepts: (1) presently, the worldwide BSE prevalence is in the trend of decline; (2) this risk assessment is essentially different from the rest of the BSE-related risk assessments previously conducted by the FSC, in that the assessed countries are only those that have not previously reported BSE cases; (3) previous risk assessments of beef and beef products from the U.S.A. and Canada were conducted by comparing their risks with that of Japanese beef and beef products so that the assessment was based on the relativity; and (4) it was foreseen to be based on the data submitted by each assessed country on a voluntary basis. Subsequently, assuming that there may be certain limitations concerning data availability and submission, the PEC decided to largely conduct this assessment on a qualitative basis but to strive to make it as quantitative as possible.

It was with this background that the PEC firstly developed an alternative assessment method suited to the current situation and then carried out BSE risk assessment for imported beef and beef offal according to this method. In this review, the authors describe the structure and logic of this assessment method. A sample assessment result is provided at the end of this article to enhance readers' understanding.

\section{PRINCIPLES OF THE CURRENT RISK ASSESSMENT}

The methodology for the current risk assessment was developed based on the previously used models for risk assessments of Japanese domestic beef and for US/Canadian beef imported into Japan [5, 6]. OIE's risk assessment criteria for BSE status and the EFSA GBR method were also referred to $[8,11]$. The PEC for the current assessment aimed 
to deliver the overall conclusion as a science-based comprehensive assessment defined by time periods and based on a combination of the following risk aspects: (1) periodic BSE risk status among the cattle population of a country, which consisted of combined risks of BSE invasion by imported live cattle and MBM, and domestic stability (inversed risk of BSE propagation), of which the latter included implementation of a feed ban and establishment of preventive measures against cross-contamination, etc., and (2) present risks of beef and beef offal processing lines, i.e., risks based on types of slaughtering and meat production processes, etc.(Fig. 1).

The current assessment was conducted on a qualitative basis rather than a quantitative basis because of the limited data regarding BSE risks. In case the data were insufficient, assessment was based on the worst-case scenario. In addition, a few cases of atypical BSE, which is biologically and biochemically different from typical BSE, have been recently found in Europe, Japan and the U.S.A. among other countries. Those cases were distinguished from the classical type of BSE mainly by band patterns of $\mathrm{PrP}^{\mathrm{Sc}}$ proteins demonstrated by Western blotting. The origin of atypical BSE is still unknown to this date, and information about BSE prion distribution in bovine tissue is scarce $[2,3]$.

Due to the above-mentioned situation, therefore, the current risk assessment was conducted with the assumption that (1) the first case of BSE occurred in the U.K. for an unknown reason, with BSE agents then being propagated through $\mathrm{MBM}$ recycling from BSE-infected cattle, and that (2) BSE infection was spread to other countries by exportation and utilization of BSE-infected live cattle and BSE-contaminated MBM for animal feeds.

\section{PERIODIC BSE RISK STATUS AMONG THE CATTLE POPULATION OF AN ASSESSED COUNTRY}

Assessment of invasive BSE risk: For the purpose of analysis in this section, the PEC defined a country in categories III or IV according to the EFSA GBR or a country with at least one BSE-positive case reported among its domestic cattle in the past as a "BSE risk country". Invasive BSE risk was assessed based on their records of live cattle, MBM and animal oil/fat importation from the BSE risk countries defined by this description.

The determined BSE risk countries were divided into the following subgroups by the level of BSE contamination as follows: the U.K., European countries with moderate contamination, European countries with low contamination, the U.S.A., Canada and others (Japan, Mexico, Chile, etc.).

Accordingly, each assessed country exporting beef and beef offal to Japan was requested to submit data regarding imports of live cattle and MBM from the BSE risk countries. Portugal had been categorized as a level IV country by the FESA GBR together with the U.K. and thus should not be grouped with other moderate-risk European countries. Nevertheless, such distinction was not made because no assessed country had a record showing importation from Portugal.

Submitted information was analyzed for possible use of the imported live cattle and MBM for animal feed produc- tion in the assessed country. In the case that the records submitted by the assessed country indicated any degree of possibility of live cattle and MBM imports from BSE risk countries having been used for animal feed, the degree of invasive BSE risk in the assessed country was estimated as a sum based on contamination probability points (CPP) of each BSE risk country. The assessment was based on a 5-year period, as this was considered to be the general term for BSE incubation.

Risks of animal oil and fat varied depending on the products' grades such as yellow grease, fancy tallow, etc., but their risks were generally regarded as low compared with that of live cattle or MBM. Thus, the information associated with animal oil/fat and their usages were taken into consideration only when importation of large quantity was recorded from BSE risk countries. Otherwise, the data were used as supplementary information.

Contamination probability points (CPP): All imported live cattle and MBM, in principle, have a potential to be used for animal feed manufacturing, but when a country could provide a feasible explanation for not utilizing any of the imported live cattle or MBM for animal feed, they could be determined as carrying no risk and thus excluded from consideration of risks.

When the track records of the imported live cattle and MBM in the assessed country showed any of the following destinies, they were regarded as adding no risk to the assessed country: (1) imported live cattle were already dead and disposed of by burial or incineration; (2) imported live cattle were still alive at the time of investigation, so they were excluded from potential use for animal feed manufacturing beforehand; and (3) imported live cattle and MBM were recorded to have been re-exported to other countries.

In this assessment, the PEC defined the invasive BSE risk as combined CPPs of imported live cattle and MBM. Its assessment was to be calculated based on the assumption that 1 ton of MBM was equivalent of 1 live bovine animal, as has been stated in the GBR by the Scientific Steering Committee (SSC) and EFSA [10].

The risks of imported live cattle and MBM from the BSE risk countries varied depending on the country and timing of importation. To reflect this variation, this assessment employed CPPs for live cattle and MBM of each BSE risk country. Records showed that the BSE prevalence in the U.K.'s live cattle was 5\% at its peak period of 1988-1993; therefore, a CPP of 1 was set as the risk of importing 1 live bovine animal from the U.K. during this period. Thus, the CPPs of the U.K. were set as shown in Table 1 based on the values indicated by the SSC's GBR and years of complete feed ban implementation in the U.K. [10].

European countries except for the U.K. were divided into two categories, namely countries of "moderate contamination" and "low contamination" [11]. The CPPs for live cattle and MBM were set up based on the SSC's GBR and years of complete feed ban implementation in European countries $[4,10]$ (Table 2). Countries such as France, the Netherlands, Belgium, and Italy were likely countries to have re-exported MBM from the U.K. and thus were given a CPP of 0.1 until 
Table 1. Periodic CPPs of live cattle and MBM from the U.K.

\begin{tabular}{|c|c|c|c|}
\hline \multicolumn{2}{|l|}{ Live cattle } & \multicolumn{2}{|l|}{ MBM (1 ton) } \\
\hline 1987 and years before & 0.1 & & \\
\hline $1988-1993$ & 1 & $1986-1990$ & \\
\hline 1994-1997 & 0.1 & 1991-1993 & 0.1 \\
\hline 1998-2005 & 0.01 & 1994-2005 & 0.01 \\
\hline 2006 and years after & 0.001 & 2006 and years after & 0.001 \\
\hline
\end{tabular}

the U.K. banned exportation of MBM (years of 1986-1996).

In the previous risk assessments done by the PEC of the FSC in Japan concerning U.S.A./Canadian beef imported into Japan, the surveillance-based BSE prevalence of U.S.A. and Canadian cattle were estimated to be 1 case and 5-6 cases per one million cattle in the U.S.A. and Canada, respectively [6]. Accordingly, the CPPs of live cattle and MBM in those two countries were set. The values are given for the periods defined by estimated year of birth among BSE-positive cattle (Table 2).

In the previous risk assessment done by the PEC of the FSC concerning U.S.A./Canadian beef imported into Japan, the surveillance-based BSE prevalence of Japanese cattle were estimated to be 5-6 cases per one million cattle. Birth years of BSE-positive cattle and the year of feed ban implementation were also taken into account to set the following CPPs for live cattle and MBM of Japan [6] (Table 2).

The CPPs for countries with no reported BSE cases could not be set by the above-mentioned BSE prevalence-based method. Since those countries were generally considered to have low BSE risks compared with countries with BSE-positive cases, CPPs were not determined for these countries. In the case that an assessed country imported a large quantity of live cattle and/or MBM from BSE-negative and GBR III countries, the information was taken into consideration as a supplementary factor for the assessment.

Assessment for invasive BSE risk: Based on the principles above, total invasive BSE risk (a sum of the invasion risks from imported live cattle and MBM) was estimated for each assessed country for a period of five years. The assessment was finally given in 5 levels, high, moderate, low, very low and negligible, as shown in Table 3.

Domestic Stability (inversed risk of BSE propagation of a country). Principles of domestic stability assessment: The essential countermeasures against BSE exposure/propagation consisted of (1) implementation of a feed ban, (2) control of SRM use, (3) optimization of rendering conditions and (4) establishment of preventive measures against crosscontamination for feed production.

Previous epidemiological analyses indicated that the most effective measure implemented in Europe was feed ban. Thus, an essential part of BSE exposure/propagation prevention was to abolish feeding of cattle with possibly BSE-contaminated MBM through animal feeds. It is in this context that a feed ban has been implemented in countries as a preventive measure against BSE. At the pragmatic level, the most effective way was to ban recycling of animal proteins regardless of animal types among mammals (ban from
Table 2. CPPs of live cattle and MBM from various countries

\begin{tabular}{|c|c|}
\hline \multicolumn{2}{|c|}{ European countries with moderate contamination ${ }^{\text {a) }}$} \\
\hline 1986-2005 & 0.01 \\
\hline 2006 and years after & 0.001 \\
\hline \multicolumn{2}{|c|}{ European countries with low contamination ${ }^{\text {b) }}$} \\
\hline $1986-1990$ & 0.001 \\
\hline 1991-2005 & 0.01 \\
\hline 2006 and years after & 0.001 \\
\hline \multicolumn{2}{|l|}{ U.S.A. } \\
\hline $1993-$ & 0.00002 \\
\hline \multicolumn{2}{|l|}{ Canada } \\
\hline $1989-$ & 0.0001 \\
\hline \multicolumn{2}{|l|}{ Japan } \\
\hline 1992-2006 & 0.0001 \\
\hline 2007 and years after & 0.00001 \\
\hline
\end{tabular}

a) France, Netherlands, Belgium, Italy, Ireland, Germany, Spain, Swiss, etc. b) Poland, Denmark, Austria, Czec Republic, Slovenia, etc.

Table 3. Total invasive BSE risk

\begin{tabular}{lc}
\hline Levels for risk of invasion & U.K. equivalent $(\mathrm{N})^{\text {a) }}$ \\
\hline High & $100 \leq \mathrm{N}$ \\
Moderate & $20 \leq \mathrm{N}<100$ \\
Low & $10 \leq \mathrm{N}<20$ \\
Very low & $5 \leq \mathrm{N}<10$ \\
Negligible & $0 \leq \mathrm{N}<5$ \\
\hline
\end{tabular}

a) Calculated based on the assumption of 1 ton of MBM equals 1 live bovine animal.

mammals to mammals), followed by less but still effective measures such as a ban on protein recycling from mammals to ruminants and then from ruminants to ruminants.

Other measures that were also indicated as important for BSE control in these analyses included exclusion of SRM from rendering materials, optimization of rendering conditions (not less than $133^{\circ} \mathrm{C}$ for a minimum of $20 \mathrm{~min}$ at an absolute pressure of 3 bar), dedication of feed mills to a single species and production line separation.

It has been stated that $99 \%$ or more of infectivity in BSEpositive bovine animals is distributed to the bodily regions called specified risk material (SRM; e.g., brain, spinal cord, etc.) [4]. Removal of SRM from rendering materials was considered to be important, and the best way to realize this measure was implementation of a legally-bound feed ban that prohibited the use of SRM and fallen stock in animal feed. Even diversion of SRM use from feed production to human consumption was considered to provide a certain degree of protection against BSE exposure/propagation, when coupled with avoidance of fallen stock use in animal feed.

Rendering under proper conditions could provide an effective reduction in BSE infectivity. For example, heat treatment $\left(126^{\circ} \mathrm{C}\right.$ for $\left.30 \mathrm{~min}\right)$ of a prion strain $(301 \mathrm{~V}$ strain) after passage using mice resulted in reduction of infectivity by 
$\log 1.9\left(\mathrm{ID}_{50} / \mathrm{g}\right)$ or $\log 2.7\left(\mathrm{ID}_{50} / \mathrm{g}\right)$. The scientific opinion of the EFSA is that heat treatment of BSE prion under certain conditions $\left(133^{\circ} \mathrm{C}\right.$ for a minimal of $20 \mathrm{~min}$ at bar 3$)$ effectively reduces its infectivity by 1,000 times [12], although the same rendering conditions may not be as effective as indicated above when the subject is a mixture of SRM and bones originating from BSE-infected cattle. Drawn from this data was the suggestion that, although the heat treatment recommended by the OIE appeared to be effective in reducing risks to certain extent, other measures should be combined with this rendering policy to completely prevent BSE exposure/propagation.

A previous study reported that oral administration of 0.1 , 0.01 and $0.001 \mathrm{~g}$ of BSE-infected cattle brain was capable of transmitting BSE at the rate of 7 in 15 cattle, 1 in 15 cattle and 1 in 15 cattle, respectively [13]. These data were consistent with the European field observation that even a trace amount of animal protein was enough to infect bovine animals through contaminated feeds. Therefore, simply washing of the processing lines in a feed factory was not sufficient to prevent cross-contamination; implementation of more advanced measures such as dedication of feed mills to a certain species of animals and line separation were required.

For assessment of domestic stability, the information submitted from each assessed country was firstly analyzed for the extent of feed ban implementation, and then other aspects such as use of SRM, rendering conditions and preventative measures against cross-contamination were evaluated.

The assessment also focused on the degree of legal obligation bound to each regulation. The data regarding compliance with those preventative regulations were also evaluated whenever the data were available.

Assessment of domestic stability: Based on the assessment principles described above, countries were firstly assessed for their domestic stability by rigidity of feed bans (e.g., abolishing the use of all mammalian products for mammalian feed, mammalian products for ruminant feed or ruminant products for ruminant feed regulations [(1)-(4) in Fig. 2]). Secondly, regulation of SRM usage was considered (○-O). In the case that SRM was used for feeds, rendering conditions and avoidance of cross-contamination were considered. Risk reduction measures such as rendering at $133^{\circ} \mathrm{C}$ for $20 \mathrm{~min}$ at $3 \mathrm{bars}$ and/or prevention of cross-contamination at feed factories, e.g., isolating production lines or establishing exclusive feed production facilities were also taken into account ( $\bigcirc$ or $\triangle$ ). Finally, the domestic stability of each country was assessed by categorizing them into one of 5 ranks, very stable, stable, middle, unstable and very unstable, based on a certain period defined by significant events such as regulatory modification.

Assessment of overall BSE risk in a cattle population, aided by verification with surveillance: Countries with high BSE propagation risks presumably bear a high risk of domestic BSE exposure/propagation upon entrance of BSE agents into the country. Thus, when the submitted data indicated a high risk of domestic BSE exposure/propagation (namely, combined risks of high invasive BSE risk and unstable domestic stability), this aspect was taken into consideration as an additional factor. In the case that an assessed country was categorized in a high exposure/propagation period at a certain time in the past, that country was assigned to a one-rank higher overall risk group for the next 5 years.

Surveillance was the essential method to scientifically verify the risk assessment output, and so surveillance data obtained from an assessed country was used for the results of the current assessment. The actual verification process was constructed based on the OIE point system (BSurvE method), as no other alternatives were available at that time.

\section{RISK ASSESSMENT OF THE PROCESS OF BEEF AND BEEF OFFAL PRODUCTION}

Ensuring the removal of SRM can remarkably reduce the risk of variant Creutzfeldt-Jakob disease (vCJD) in humans. Therefore, this measure is currently at the center of preventive policies regarding protection of human and cattle health from BSE. The current risk assessment for beef and beef offal firstly evaluated the extent of "SRM removal" done by each country, followed by combined assessment of items such as "inspection at slaughterhouses" and "stunning/pithing" to evaluate the risk-reducing efficiency of meat processing lines.

SRM removal: In a BSE-positive bovine animal, 99\% or more of its infectivity is attributed to SRM [9]. Thus, exclusion of these materials from the food chain ensures reduction in most of the vCJD-associated risks in humans. To reflect these SRM-related aspects, the current assessment also took into consideration factors such as implementation of SRM removal or any other measures in preventing beef and beef offal from being contaminated by BSE agents. This part of the assessment was ultimately designed to determine whether cross-contamination preventive measures and efficacy verification systems for them had been established in each country.

The definition of SRM adopted in this assessment was from the OIE's SRM definition for a "controlled risk country" based on the following reasons: (1) the currently assessed countries all had no BSE-positive reports; (2) there were, however, some countries that were categorized into GBR III of the EFSA; and (3) the definition and handling of SRM varied among the assessed countries. When the SRM definition of the assessed country was acknowledged to be largely different from that of the OIE, the assessment was conducted in a manner separate from these general principles and handled on a case-by-case manner.

Inspection, stunning and pithing at slaughterhouses: Elimination of high risk cattle such as downer cows is an important protocol in protecting human health from BSE risks, and for this reason, the OIE code requires proper antemortem inspection before slaughtering [9]. However, it is also known that clinical observation for possible symptoms alone is not enough to distinguish BSE-infected cattle from other diseases. Therefore, both the provisions of (1) effective elimination of downer cows at the antemortem inspection and (2) BSE testing at slaughterhouses were evaluated in the current assessment. 


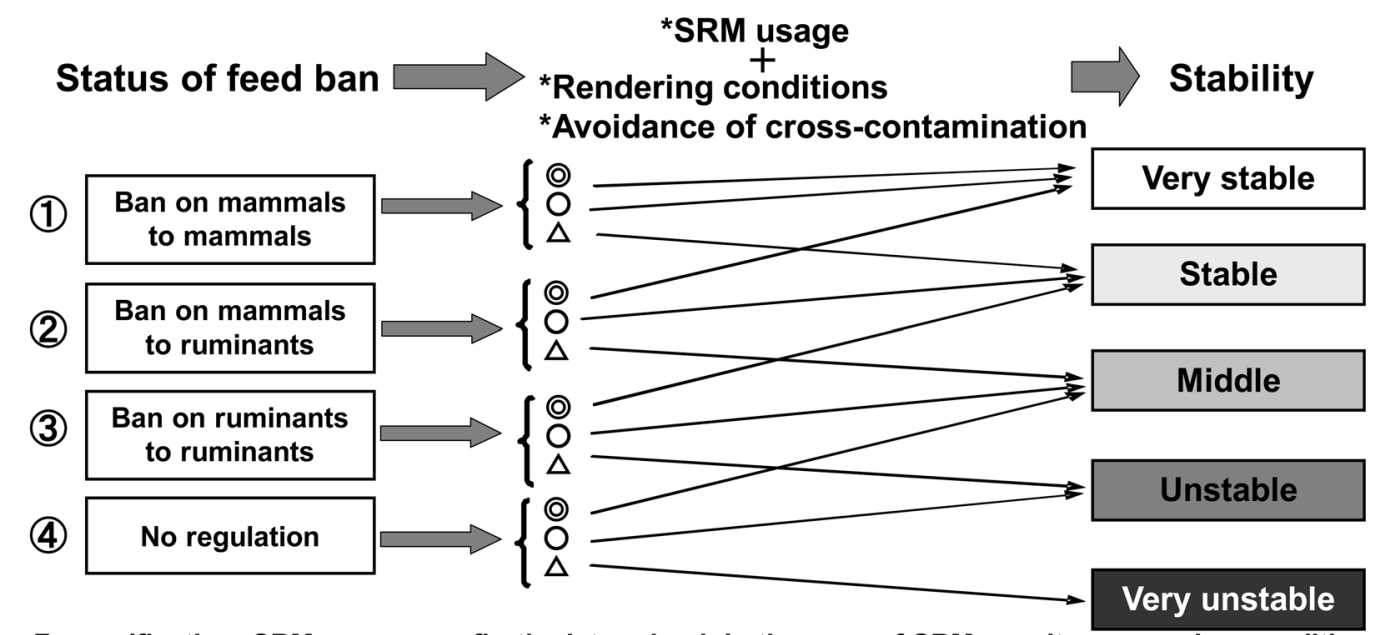

* For verification, SRM usage was firstly determined. In the case of SRM use, its processing conditions such as rendering conditions and measures against cross-contamination were assessed.

1. Regulations on SRM usage

\begin{tabular}{|l|c|}
\hline \multicolumn{1}{|c|}{ Measures } & Rating \\
\hline $\begin{array}{l}\text { - Ban on use of SRM and fallen } \\
\text { stock }\end{array}$ & 0 \\
\hline $\begin{array}{l}\text { - Ban on use of fallen stock } \\
\text {-SRM is used for other than feed }\end{array}$ & 0 \\
\hline -SRM is used for feed & $\begin{array}{c}\text { Proceed } \\
\text { to } 2 .\end{array}$ \\
\hline
\end{tabular}

2. Rendering conditions and

preventative measures against cross-contamination

\begin{tabular}{|l|c|}
\hline \multicolumn{1}{|c|}{ Measures } & Rating \\
\hline -All rendering facilities: $133^{\circ} \mathrm{C} / 20 \mathrm{~min} / 3$ bars & \\
and/or & \\
-Prevention of cross-contamination & 0 \\
(Separate production lines, exclusive & \\
facilities) & \\
\hline -Others & $\Delta$ \\
\hline
\end{tabular}

$\checkmark$ Terms were defined by significant events in association with regulatory measures.

Example: \begin{tabular}{|l|ll|l|rr|}
\hline Domestic stability & \multicolumn{3}{c|}{ Middle } & Stable & \multicolumn{2}{c|}{ Very stable } \\
\hline
\end{tabular}

Fig. 2. Assessment of domestic stability. Each country was evaluated by the range of subjected animals and rigidity of the feed ban, followed by SRM-related conditions, to be assigned to one of the 5 ranks indicating degree of domestic stability. The rigidity of feed ban was rated based on the strengths of SRM-handling measures (the two lower boxes). At the bottom, an example of domestic stability is shown per terms defined by significant regulatory changes.

Pithing of animals at the slaughterhouse is linked to an increased risk of BSE contamination via brain and spinal tissue spillage from the stunning hole onto the processed meat and slaughtering facilities. It also increases the possibility of high-risk tissue (brain and spinal cord) leakage into the blood stream. Likewise, a stunning method with intracranial air/gas pressuring may also bring about a similar manner of contamination [1]. Therefore, the current assessment took into consideration slaughtering processes such as implementation of pithing or air/gas injection stunning in each assessed country.

Others (mechanically recovered meat, MRM, etc.): MRM including meat from advanced meat recovery (AMR) is meat obtained from bones by secondary recovery using mechanical techniques after primary removal of the major meat blocks. This method carries a certain risk of SRM contamination. Thus, the same assessment method as for primary beef meat blocks cannot be adopted for MRM in evaluation of the risk-reducing efficacy of BSE measures. Accordingly, the Commission requested the assessed countries known for MRM production to submit additional information regarding the SRM definition, measures and MRM exportation to Japan, and then these data were assessed separately from general beef and beef offal exports.

The total number of livestock and their traceability were also requested as supplementary data because they are related to the sensitivity and precision of antemortem inspection at the slaughterhouse or estimation of animal age in months.

Overall assessment of risk-reducing measures at meat processing lines: The process of assessment for efficacy of risk reduction during meat processing is concisely demonstrated in Fig. 3, aided by the explanation as follows. The efficacy of risk reduction during meat processing was assessed firstly by the degree of SRM removal. In the case that SRM removal was conducted in accordance with regulations, the risk reduction level was regarded as the highest, followed by voluntary removal; no regulation was the worst grade. Secondly, the actual measures used for SRM removal were 


\section{Removal of SRM*1}

2. BSE testing, pithing and stunning status

\section{\begin{tabular}{l|}
\hline SRM removal \\
required by law ${ }^{* 2}$
\end{tabular} \\ SRM removal on a voluntary basis}

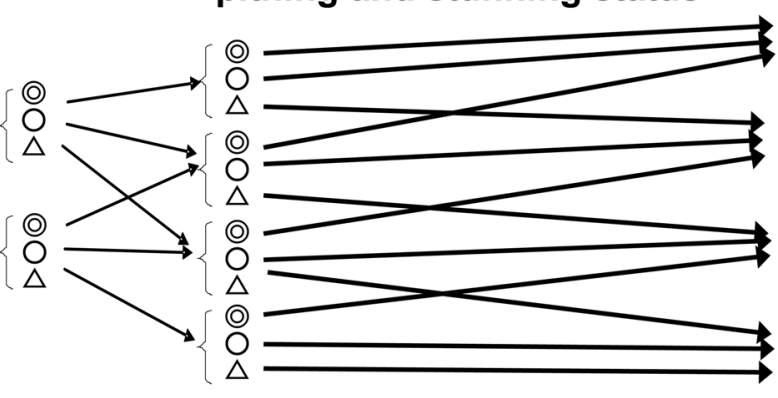

No SRM removal
3.Risk reduction efficacy at slaughterhouse

Extremely effective

Highly effective

Moderate level

Low level

*1 Judgment was based on the conditions of SRM removal and procedures at slaughterhouses (If available, data of actual compliance was also taken into consideration.)

*2 Removal by domestic regulation, or by additional conditions required for exportation to Japan

\section{SRM removal}

1. SRM removal Measures
\begin{tabular}{|l|c|}
\hline \multicolumn{1}{|c|}{ Rating } \\
\hline (1) Confirmation by meat inspector & \\
(2) Carcasses washed by high pressure water & \\
(3) Washing of the saw between carcasses & () \\
(4) SSOP and HACCP regulations & \\
3 or 4 of the above measures implemented & \\
\hline 2 of the above measures implemented & $\bigcirc$ \\
\hline Others & $\triangle$ \\
\hline
\end{tabular}

\section{BSE testing, stunning and pithing status}

\begin{tabular}{|c|c|}
\hline Measures & Rating \\
\hline $\begin{array}{l}\text { - Health inspection to eliminate downers } \\
\text { and BSE testing at slaughterhouses } \\
\text { and } \\
\text {-Ban on air pressure stunning and pithing } \\
\text { Both measures implemented }\end{array}$ & (2) \\
\hline One measure implemented & $\mathrm{O}$ \\
\hline Others & $\triangle$ \\
\hline
\end{tabular}

Fig. 3. Flow chart of the assessment for efficacy of risk reduction at slaughterhouses. Each county was firstly reviewed for the status of SRM removal, followed by the rating of other risk-avoiding measures, to determine its risk reduction efficacy.

verified based on the actual conditions for such things as meat inspector's process confirmation, washing of carcasses with high pressure water to assure the complete removal of central nervous tissues, washing of the saws between individual carcasses and regulation by a Sanitation Standard Operating Procedure (SSOP) and Hazard Analysis and Critical Control Points (HACCP). If 3 or 4 of the above measures were conducted, the risk reduction efficacy was given a one-rank promotion when proceeding to the next assessment point (๑). The second verification point for risk reduction was the combination of BSE testing and absence of pithing/ air stunning. If health inspections to eliminate downers and BSE testing were conducted in the slaughterhouse together with bans for both air pressure stunning and pithing, the efficacy of risk reduction was promoted one rank (๑). If one of the above measures was conducted, the efficacy of risk reduction stayed at the same level (O);otherwise, the country was demoted one rank. Based on the above-mentioned principles, each assessed country was categorized according to 5 grades for the efficacy of its risk-reducing measures, namely no effect, low level, moderate level, highly effective and extremely effective (Fig. 3).

Since the current assessment subjected only the meat products imported by Japan, the scope of evaluation was fo- cused on the criteria for beef product preparation and BSEpreventive measures intended for each country's exports to Japan. Presently, all the beef and beef offal importers in Japan are requested to voluntarily refrain from importing SRM from any foreign countries. Some exporting countries even have their own specific regulations on exclusion of SRM from beef and beef offal exports to Japan under the Animal Health Requirement requested from Japan. Therefore, these risk control measures were also taken into the assessment along with the information obtained from each country upon the Commission's request through a questionnaire.

\section{RISK OF MECHANICALLY RECOVERED MEAT (MRM)}

Among the countries that have submitted replies to Japan's inquiry, two countries reported domestic production of MRM, with one country having exported $81.6 \mathrm{~kg}$ of MRM (head parts not included in raw materials) to Japan in 2008, while the other had no such record.

The key structure of the current risk assessment was the evaluation of imported beef and beef offal to Japan, which consists of a combination of multiple aspects such as risk of live cattle and risk-reducing measures at slaughterhouse 
and meat processing lines in fabrication plants. Accordingly, any commodities that do not fall into these categories, such as MRM, should be considered separately. There are so far no grounds to suggest that MRM contamination is not connected with SRM through meat processing lines. Thus, at least MRM from those countries that have potentially had exposure to and/or propagation of BSE in the immediate past according to the data collected should be regarded as carrying certain risks. However, MRM from countries that are regarded as having a negligible possibility of BSE exposure/ propagation may be considered as carrying negligible risks, provided the precondition of the current assessment, namely classical BSE originating from the U.K., is appropriately met.

Recently, there have been a few cases of irregular forms of BSE (atypical BSE) reported apart from classical BSE in Europe, Japan and the U.S.A. These reports of atypical BSE indicated variation in molecular sizes of abnormal prion proteins (PrPSc) among cases, and eventually two major sizes of proteins were designated as the $\mathrm{H}$ and $\mathrm{L}$ types.

Most of the atypical BSE cases were found in aged cattle over 8 years old, but a remarkable exception exists in Japan, where a steer only 23 months old was reported to have been infected with atypical BSE (the 8th BSE case in Japan). When this exception was excluded, the detection ages of atypical BSE cases ranged from 6.3 to 18 years old. The average detection ages for the $\mathrm{H}$ and $\mathrm{L}$ types were 11.8 and 11.6 years old, respectively [3].

To the best of the authors' knowledge, there have been about 40 cases of atypical BSE reported worldwide, yet the OIE does not require distinction between classical and atypical BSE cases in member countries for their reports, while the EFSA only recently referred to case reporting by classical/atypical recognition in its 2009 scientific opinion. These situations seem to further obscure the clear number of atypical BSE cases occurring in the world.

The origin of atypical BSE has not yet been determined. According to EFSA's scientific opinion published in 2008, all the cases of atypical BSE were reported with birth dates before the real feed ban in January 2001 in Europe. Therefore, the possibility of these atypical cases being attributed to contaminated feeds, just as in classical BSE, cannot be completely denied. On the other hand, data of atypical BSE cases (both the $\mathrm{H}$ and $\mathrm{L}$ types) in France did not show any reasonable correlation between birth year and frequency of occurrence, as was indicated in classical BSE cases, thus raising the possible interpretation of atypical BSE being sporadic isolated cases of prion disease [3].

Based on the data accumulated in France, the frequencies of atypical BSE cases per 1 million tested adult cattle were estimated to be 0.41 and 0.35 cases for the $H$ and $L$ types, respectively (1.9 and 1.7 cases for the $\mathrm{H}$ and L-types, respectively, when limiting the sampling to tested cattle over 8 years old). In Japan, a total of 10 million cattle including fallen stock and slaughtered cattle were tested for BSE, and the results showed no positive cases of the $H$ type and 2 positive cases (case 8 , a 23 -month-old steer; case 24 , a 169-month-old Japanese black cow) of the L type of atypical
BSE. These data indicate that Japan has prevalence frequencies of 0 and 0.2 cases of the $H$ and L types of atypical BSE per 1 million cattle including tested fallen stock and slaughtered cattle (zero and approximately 1.5 cases of the $\mathrm{H}$ and $\mathrm{L}$ types respectively, when limiting the sampling to tested slaughtered cattle over 8 years old).

Atypical BSE of both the $\mathrm{H}$ and $\mathrm{L}$ types was confirmed to be transmissible by intracerebral inoculation in transgenic mice expressing alleles of bovine or ovine PrP genes and of inbred mice. However, for transgenic mice expressing human prion protein, the $\mathrm{L}$ type but not the $\mathrm{H}$ type could be transmitted according to the previously published reports (recently, it was reported that $\mathrm{H}$ type also transmissible to the humanized transgenic mice). There have also been reports of glycosylation pattern transformation from L-type BASE3 $\mathrm{PrP}^{\mathrm{Sc}}$-like type to more of the classical BSE PrPSc type. This phenomenon was observed during passage using inbred and transgenic TgVR2 mice. As for the atypical cases of BSE confirmed in Japan, the 24th case of BSE was determined to have had the atypical L type at the detection age of 169 months old, and its sample was successfully transmitted to transgenic mice expressing bovine prion protein. However, transmission of a sample from the other case of atypical L-type BSE confirmed in Japan (the 8th case; detected at the age of 23-month-old) was reported to be unsuccessful in transgenic mice expressing bovine prion protein. The reason for this inconsistency is not clear at this time, although the possible presence of a limitation in the amount of prion protein accumulated in the subject's brain sample or that the inoculated volume was too low to reach the detection limit cannot be excluded.

A recent report has shown that the atypical L type of BSE has a higher degree of potential for pathogenicity than that its classical counterpart because incubation periods are shorter in atypical BSE transmitted to transgenic mice expressing human prion protein, suggesting that atypical BSE possibly has a higher degree of pathogenicity when compared to its classical counterpart [7].

In contrast to classical BSE, the systemic distribution of abnormal prion protein in atypical BSE cases is barely known. Therefore, it is unclear whether the brainstem is truly the optimal part for sampling and testing in $\mathrm{H} / \mathrm{L}$ type detection. Likewise, information regarding the infectivity distribution of atypical BSE is scarce in bovine peripheral tissues and body fluid. All together, the lack of essential data hinders, to a certain extent, evaluation of the relative risk-reducing effects of various SRM removal measures for cattle.

Based on the currently available data concerning the potential risks for humans of atypical BSE and prevalence of atypical BSE, it may be too extreme to deny the risk of MRM, especially in MRM derived from aged cattle. However, the degree of influence of the presence of atypical BSE on our concept of the MRM risk will be limited to a low level under the circumstances with presently available knowledge and our discussion. In the meantime, one must also be reminded of the fact that only a limited amount of data is currently available concerning atypical BSE. A proper amount of dis- 

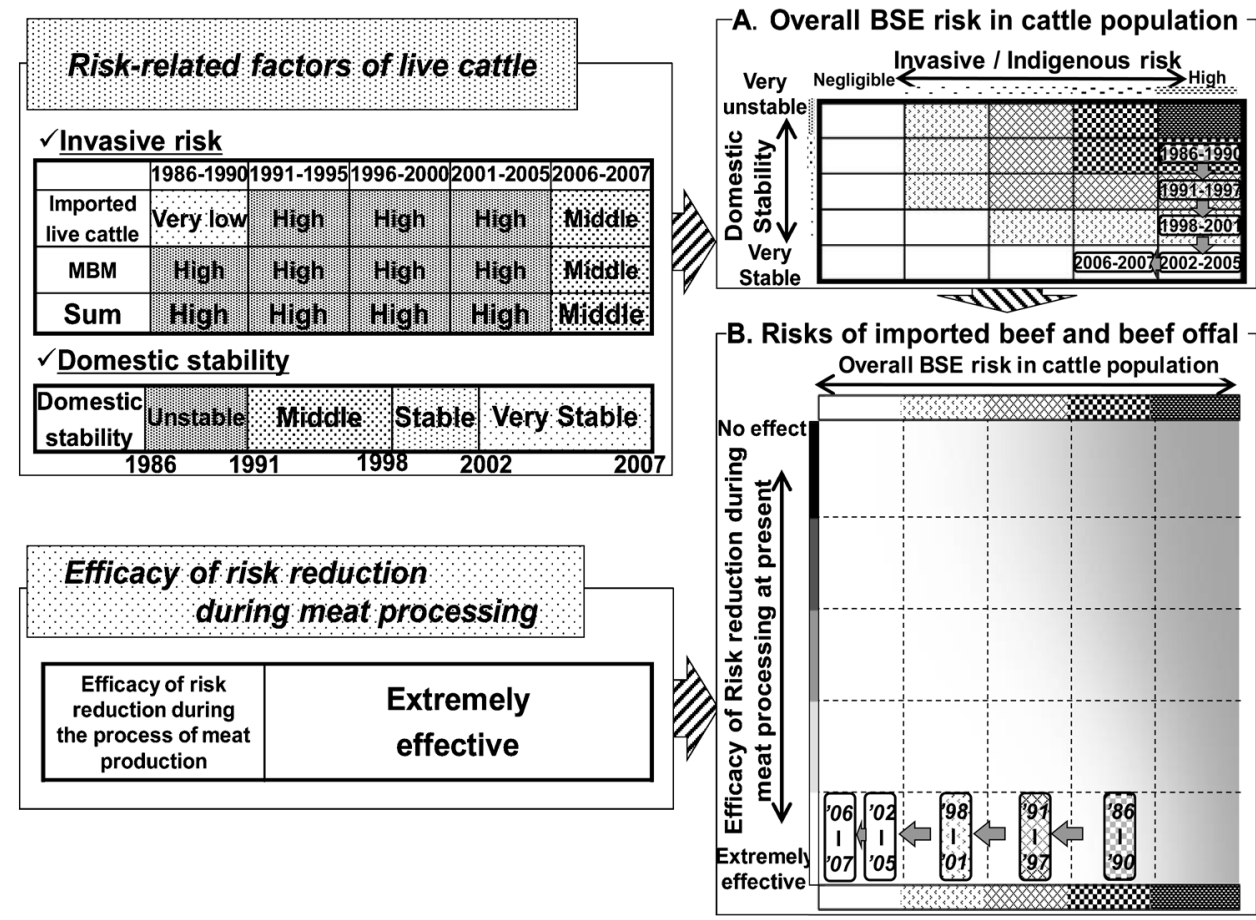

Fig. 4. Overall assessment results in a schematic view. A: The shift of overall BSE risks among age cohorts. The total invasive risks (the sum of imported live cattle and MBM risks; vertical axis) were plotted against domestic stability ranks (horizontal axis) in 2-D rank plot A. Note that the horizontal axis partially refers to the indigenous risk (domestic BSE propagation), which is also a reflection of invasive risk and domestic stability. Therefore, the results, shown in rank plot A, indicate the shift of BSE infection risks among the birth cohorts. B: The age-specific BSE risks in rank plot A and the efficacy of risk reduction during meat processing were combined. The outcome, 2-D rank plot B, was used to supplement the assessment process to get the final conclusion for each assessed country.

cretion should be used when interpreting these data to avoid unnecessary confusion. Further research and accumulation of data will bring additional insight into the mechanism, pathogenicity and transmission potential of atypical BSE, for which further assessment may become necessary in the future.

\section{RISK ASSESSMENT OF BEEF AND BEEF OFFAL IMPORTED INTO JAPAN}

To gain the final result of this assessment, the periodic BSE risk status of a country (the sum of invasive BSE risk and domestic stability) and efficacy of present BSE riskreducing measures at meat processing lines were combined and used as an indicator of comprehensive likelihood of BSE prion contamination in beef and beef offal imported into Japan. Surveillance data were used to verify reliability of the assessment. Finally, a summary of each country was expressed in schematic figures (an example is shown in Fig. 4).

In Fig. 4, a model country's invasive risk was ranked as high (from 1986-2005) but was reduced to the middle level from 2006 onwards. The efficacy of feed ban (domestic stability) was unstable during 1986-1989 but improved to the middle level (1990-1996), to the stable level (1997-2000) and then to the very stable level (2001-until now). Current risk reduction efficacy at meat processing lines, determined by factors such as the definition of SRM, compulsory removal of SRM by law, and HACCP/SSOP procedures were good and verified and were therefore rated as $\odot$. BSE testing at slaughterhouse ( $>30$ months), proper slaughtering procedures such as avoidance of air stunning and pithing were verified as $\odot$. All together, the overall risk reduction was extremely effective.

The final assessment for this model country was as follows: the domestic BSE exposure/propagation risk was low, and risk reduction at meat processing lines was extremely effective; therefore, the risk of BSE contamination of beef and beef offal imported from this assessed country was considered to be negligible.

ACKNOWLEDGMENTS. This work was conducted by the PEC of the FSC in Japan. The members of the PEC appreciate the FSC staff for their laborious efforts in data collection and processing, Dr. Ruriko Nakao for her assistance in manuscript preparation and the FSC counterparts of the assessed counties for their data contribution. Without their sincere help, we could not complete this work. 


\section{REFERENCES}

1. Anil, M. H., Love, S., Williams, S., Shand, A., McKinstry, J. L., Helps, C. R., Waterman-Pearson, A., Seghatchian, J. and Harbour, D. A. 1999. Potential contamination of beef carcases with brain tissue at slaughter. Vet. Rec. 145: 460-462. [Medline] [CrossRef]

2. Biacabe, A. G., Morignat, E., Vulin, J., Calavas, D. and Baron, T. G. 2008. Atypical bovine spongiform encephalopathies, France, 2001-2007. Emerg. Infect. Dis. 14: 298-300. [Medline] [CrossRef]

3. Ducrot, C., Arnold, M., de Koeijer, A., Heim, D. and Calavas, D. 2008. Review on the epidemiology and dynamics of BSE epidemics. Vet. Res. 39: 15. [Medline] [CrossRef]

4. European Food Safety Authority. 2007. Opinion of the Scientific Panel on Biological Hazards on the revision of the Geographical BSE risk assessment (GBR) methodology. EFSA J. 463: 1-35.

5. Food Safety Commission of Japan. 2004. Measures against Bovine Spongiform Encephalopathy (BSE) in Japan (Interim report). Available from http://www.fsc.go.jp/sonota/measure bse_injapan.pdf

6. Food Safety Commission of Japan. 2005. Risk assessment concerning "the comparability between risks of consuming beef and internal organs regulated by the beef export verification program of the United States/Canada and risks of consuming beef and internal organs of Japanese cattle". Available from http://www. fsc.go.jp/sonota/bse-risk-assessment-concerning.pdf

7. Iwamaru, Y., Imamura, M., Matsuura, Y., Masujin, K., Shimizu, Y., Shu, Y., Kurachi, M., Kasai, K., Murayama, Y., Fukuda, S., Onoe, S., Hagiwara, K., Yamakawa, Y., Sata, T., Mohri, S.,
Okada, H. and Yokoyama, T. 2010. Accumulation of L-type bovine prions in peripheral nerve tissues. Emerg. Infect. Dis. 16: 1151-1154. [Medline]

8. L'Office International des Épizooties. 2004. Terrestrial Animal Health Code 2004. BSE Chapter of the OIE Terrestrial Animal Health Code, 1.3. Categorisation of countries according to the BSE risk. Available from http://www.oie.int/doc/ged/D6450. PDF

9. L'Office International des Épizooties. 2008. Terrestrial Animal Health Code 2008. VOLUME II CHAPTER 11.6. Available from http://www.oie.int/doc/ged/D6436.PDF

10. Scientific Steering Committee of European Commission. 2000. Final opinion on the Geographical Risk of Bovine Spongiform Encephalopathy (GBR). Available from http://ec.europa.eu/ food/fs/sc/ssc/out113_en.pdf

11. Scientific Steering Committee of European Commission. 2002. Update of the Opinion of the SCIENTIFIC STEERING COMMITTEE on the Geographical Risk of Bovine Spongiform Encephalopathy (GBR). Available from http://ec.europa.eu/food/ fs/sc/ssc/out243_en.pdf

12. Taylor, D. M. 2000. Inactivation of transmissible degenerate encephalopathy agents: a review. Vet. J. 159: 10-17. [Medline] [CrossRef]

13. Wells, G. A., Konold, T., Arnold, M. E., Austin, A. R., Hawkins, S. A., Stack, M., Simmons, M. M., Lee, Y. H., Gavier-Widén, D., Dawson, M. and Wilesmith, J. W. 2007. Bovine spongiform encephalopathy: the effect of oral exposure dose on attack rate and incubation period in cattle. J. Gen. Virol. 88: 1363-1373. [Medline] [CrossRef] 